\title{
Selection of Trypanosoma cruzi Clonal Genotypes (Clonet 20 and 39) Isolated from Bolivian Triatomines Following Subculture in Liquid Medium
}

\author{
Marie-France Bosseno, Nina Yacsik*, Fernando Vargas*, \\ Simone Frédérique Brenière/ ${ }^{+}$
}

UMR CNRS/ORSTOM No. 9926, BP 5045, 34032 Montpellier Cédex 1, France *Universidad Mayor de San Andrés, Instituto Boliviano de Biología de Altura, Casilla Postal 641, La Paz, Bolivia

Previous studies showed that two groups of Trypanosoma cruzi clonal genotypes named clonet 20 and clonet 39 were predominant in Triatoma infestans, the unique vector of Chagas disease in Bolivia. These groups of clones correspond to distinct genetic clusters. These clonets were detected in $\mathrm{T}$. infestans and Rhodnius pictipes fecal samples before isolation and after culture by kDNA PCR (polymerase chain rreaction) and hybridization of the amplified products with clonet specific kDNA probes named 20 and 39 as previously reported. Forty eight T. infestans and three R. pictipes infected insects captured at random in different Bolivian departments were proceeded. As previously reported the direct identification of the two major clonets in fecal samples allowed the detection of abundant mixed infections: $41 \%$ in the original sample, however after culture, only $6 \%$ of mixed infections were detected. Among the 21 parasite stocks isolated from digestive tracts where mixed infections were initially detected (clonet $20+39)$ clonet 20 alone was detected in $81 \%$ of them. This result clearly showed that the culture step selected clonet 20 parasites over those belonging to clonet 39. The taxonomic status of the isolated stocks was also confirmed by isoenzyme typing, and correlation was observed between clustering topology and hybridization patterns with the probes 20 and 39.

Key words: Trypanosoma cruzi - Triatoma infestans - Bolivia - clonal genotypes - culture - selection

Natural populations of Trypanosoma cruzi, the agent of Chagas disease, have basically a clonal population structure (Tibayrenc \& Ayala 1988) and consist of heterogeneous mixtures of clones. Moreover, population genetic studies showed that T. cruzi taxon appears to be composed of two major phylogenetic clusters (Souto et al. 1996, Nunes et al. 1997, Bastrenta et al. 1999) each extensively variable and probably subdivided in smaller clusters (Tibayrenc 1995, 1998).

The genetic characterization by isoenzymes and DNA analysis requires a substantial quantity of parasite. Parasite stocks are isolated directly from

\footnotetext{
This work was supported by a grant from the World Health Organization Special Program for Research and Training in Tropical Diseases (No. 910273).

${ }^{+}$Corresponding author. Fax: +52-5 622 3369. E-mail: breniere@servidor.unam. at Instituto de Investigaciones Biomédicas, Ciudad Universitaria, UNAM, Departamento de Inmunología, AP 70228, CP 04510, México DF

Received 26 May 1999
}

Accepted 23 February 2000 the digestive tract of insect vectors, and from blood of infected mammals and patients by haemoculture, xenodiagnosis and inoculation of blood into mice. The in vitro culture step is always necessary and during this amplification, a selection of clones may occur (Miles \& Cibulskis 1986, Brenière et al. 1989). The direct identification of $T$. cruzi clones or groups of clones genetically related (clonet), in feces and blood, provided a tremendous contribution to confirm the heterogeneity within an infecting population (Avila et al. 1991, Veas et al. 1991). Previously, the direct detection of clonet 20 and 39 using poymerase chain reaction (PCR) and clonet specific kinetoplast DNA probes, showed that these groups of clones, which are genetically very different, are major in the domestic Bolivian cycle (Brenière et al. 1995, 1998). Each clonet 20 and 39, belong to a different lineage of $T$. cruzi, named First and Second major clades (Tibayrenc 1998) or Lineage 2 and 1 (Souto et al. 1996) respectively. The two clonets present similar frequencies in $T$. infestans (the principal vector) and are frequently associated in a single vector (Brenière et al. 1995, Bosseno et al. 1996). In contrast, clonet 39 is more frequently detected in young chagasic Bolivian patients (Brenière et al. 1998) and adults (unpublished observations) than clonet 20. 
The purpose of this work was to compare clonets 20 and 39 frequencies in vectors before and after isolation and mass culture of parasites, in order to measure the magnitude of clonet selection during culture. The two clonets were first detected in feces by PCR (amplification of the hyper variable regions of kDNA minicircles, HVRm) and hybridization of the PCR products with clonet specific kDNA probes. Then, clonets 20 and 39 were similarly identified in the isolated stocks. Additional isoenzyme typing of the different stocks provided complementary information.

\section{MATERIALS AND METHODS}

Samples - A total of 48 T. cruzi stocks from T. infestans and three from Rhodnius pictipes captured in various Bolivian areas in domestic and peridomestic sites were isolated. The digestive tract of each insect was placed in NNN biphasic medium supplemented with $1.5 \mathrm{ml}$ of LIT medium and incubated at $28^{\circ} \mathrm{C}$ without shaking. Then subcultures of parasites were processed in small volumes of LIT medium ( $3 \mathrm{ml}$ ) until their adaptation. Mass cultures were obtained by subcultures in higher LIT medium volumes ( 10 to $50 \mathrm{ml}$ ) at $28^{\circ} \mathrm{C}$ without shaking. Finally, the adequate quantity of parasites for isoenzyme electrophoresis (20-50 mg of parasite pellet) was obtained after 4 to 6 weeks. The genetic characterization of the stocks by isoenzyme and PCR/hybridization was done from the same collection of cultured parasites.

All fecal samples presented flagellates (direct microscopical observation) and before isolation of the stocks, a drop of feces from each insect was collected in appropriate PCR conditions and preserved at $-20^{\circ} \mathrm{C}$ for further determination of the clonal composition by PCR/hybridization.

Identification of clonet 20 and 39 by PCR/ hybridization - The procedures for feces collection, extraction and PCR were according to Brenière et al. (1992, 1995), using primers (Genset, Paris, France) chosen to amplify the hyper variable region of the kinetoplast minicircles (HVRm). After electrophoresis the PCR products were transferred on to membranes and successively hybridized with the specific clonet probes 20 and 39 (Veas et al. 1991, Brenière et al. 1992). Total DNAs were purified by phenol/chloroform extraction from parasite pellets of each stock and similarly, the clonets were detected by hybridization of the PCR products amplified from total DNAs.

Isoenzyme characterization - The electrophoretic study was carried out on cellulose acetate plates, according to Ben Abderrazak et al. (1993) with slight modifications. Twelve enzymes systems were surveyed (13 enzymatic loci): glutamate oxaloacetate transaminase (GOT, EC 2.6.1.1), glucose-6-phosphate dehydrogenase (G6PD, EC 1.1.1.49), glucose phosphate isomerase (GPI, EC 5.3.1.9), glutamate dehydrogenase NAD+ (GDH NAD+, EC 1.4.1.2), glutamate dehydrogenase NADP+ (GDH NADP+, EC 1.4.1.4), isocitrate dehydrogenase (IDH, EC 1.1.1.42), malate dehydrogenase (MDH, EC 1.1.1.37), malic enzyme (ME, EC 1.1.1.40), peptidases (substrates: L-leucylleucine-leucine and L-leucyl-L-alanine) (PEP, EC 3.4.11 or $\left.13 .^{*}\right)$, 6-phospho-gluconate dehydrogenase ( PGDH, EC 1.1.1.44), and phosphoglucomutase (PGM, EC 2.7.5.1). Jaccard's distances (Jaccard 1908) were used to estimate the phenetic divergence between the stocks. The UPGMA method [unweighted pair-group method with arithmetic averages, Sneath and Sokal (1973)] was used to cluster the zymodemes according to their Jaccard's distances. The dendrogram was obtained using the Mac Dendro software computer program (Thioulouse 1989).

\section{RESULTS}

Table I summarized the results of clonet identification in feces before culture and in the corresponding stocks after mass culture. The samples (51 feces extracts and 51 DNAs from cultivated parasite) gave a PCR major band of 270 $\mathrm{bp}$, previously demonstrated to be specific for the T. cruzi species (Veas et al. 1991). Four different hybridization patterns, identical to those previously illustrated (Veas et al. 1991, Brenière et al. 1995, Bosseno et al. 1996), were observed: (1) hybridization with only probe 20 or 39 , (2) hybridization with both probes (mixed infections), (3) absence of hybridization by both probes suggesting the presence of other clone genetically unrelated to clonet 20 and 39.

Clonet distributions before and after culture were radically different $\left(X^{2}=23.7 ; \mathrm{df}=3 ; p<0.001\right)$. The frequency of clonet 39 was 0.72 in fecal samples and lower after culture $(0.35)$. The frequency of clonet 20 was identical before and after culture (0.55), but only three mixed infections were detected after culture in contrast to the important number of mixed infections observed in feces $(41 \%)$.

After culture, 11 samples exhibited clones different from those detected in the feces. This result indicated additional mixtures of clones in the original populations with as many as $60.8 \%$ of mixtures being present. Among 21 stocks isolated from triatomines with mixed infection, one gave another clone, unrelated to clonet 20 and 39 after culture, showing the presence of at least three different clones in a single vector. As previously reported, clonets 20 and 39 were frequently detected in the feces, but in the majority of the cases, only clonet 20 was identified after culture ( $76 \%$ of cases). The 
agreement of the clonal composition before and after culture was only $41.2 \%$. The discrepancy is principally caused by the deficiency of mixture detection in the isolated stocks. Moreover, a large majority of the stocks isolated from insects with mixed infection was clonet 20; this clonet was particulary selected by the culture step.

The 51 isolated stocks were analyzed by isoenzymes (13 loci) and compared with two refence stocks SO34 cl4 and MN, belonging to clonets 20 and 39 respectively. Twenty different zymodemes were iden- tified. Table II shows the genetic composition of the stocks. Only one stock presented a superposition of enzyme pattern for GPI compatible with a mixture of clones and the other patterns were identical to zymodeme 4; the PCR/hybridization of the DNA was only positive with probe 20 . The majority of the stocks presented the same or a very close zymodeme as the reference stocks ( $\mathrm{SO} 34 \mathrm{cl} 4=\mathrm{Z1}$ and $\mathrm{MN}=\mathrm{Z11})$. The dendrogram (Figure) constructed from the matrix of Jaccard's distances showed that the stocks were clustered in two upper branches, each including a

\section{TABLE I}

Modifications of Trypanosoma cruzi clonal composition before and after isolation and culture of stocks isolated from vectors

\begin{tabular}{lccccc}
\hline \multicolumn{5}{c}{ Clonets identification, no. } \\
\hline & $\begin{array}{c}\text { Before culture } \\
\text { (feces) }\end{array}$ & Clonet 20 only & Clonet 39 only & Clonet 20 + 39 & Other clones \\
\cline { 3 - 6 } & 7 & 5 & 1 & 1 & 0 \\
Clonet 20 only & 16 & 3 & 10 & 1 & 2 \\
Clonet 39 only & 21 & 16 & 3 & 1 & 1 \\
Clonet 20 + 39 & 7 & 1 & 1 & 0 & 5 \\
Other clones & 51 & 25 & 15 & 3 & 8 \\
\hline Total & & & & &
\end{tabular}

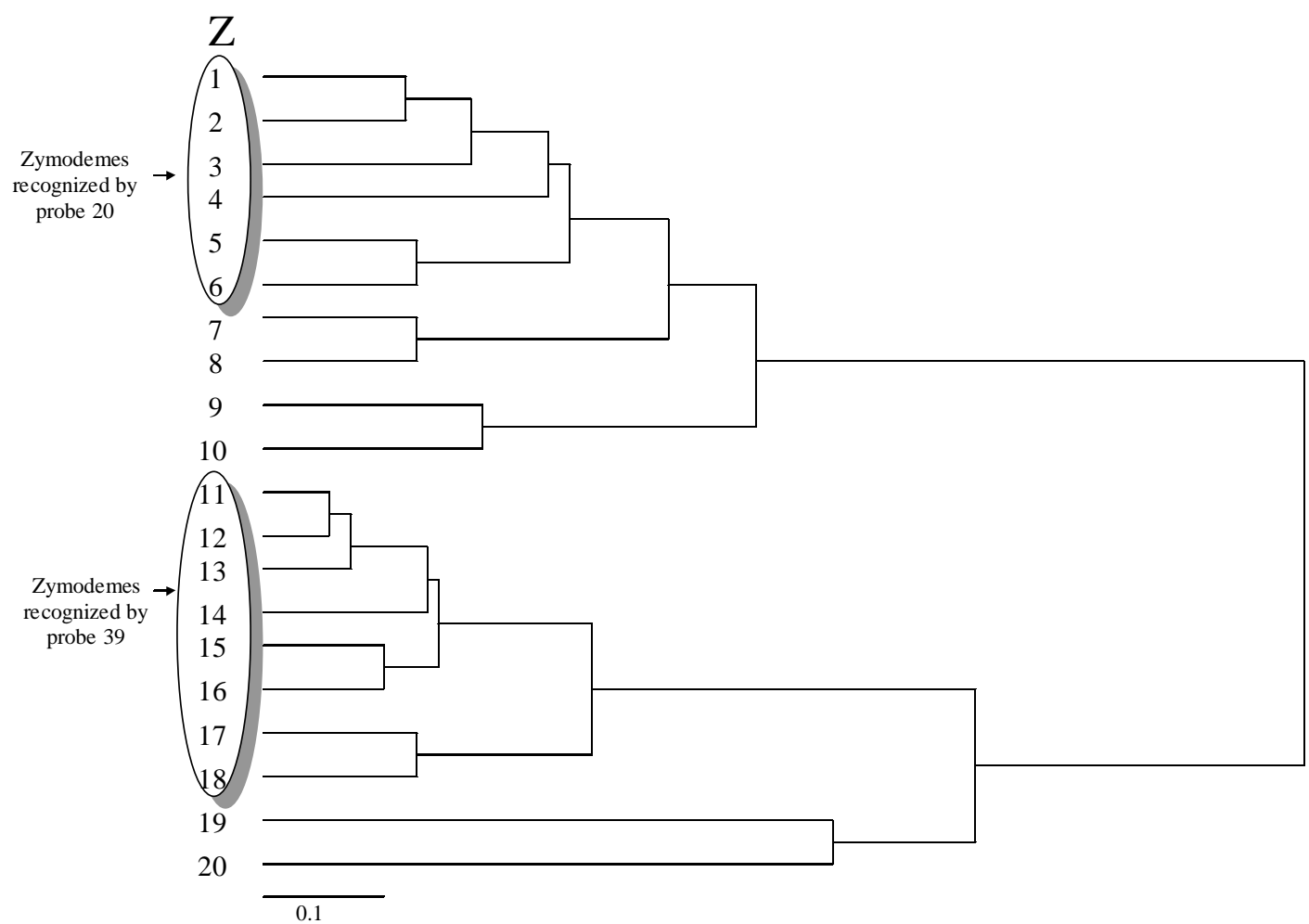

Dendrogram constructed using the unweighted pair group method with Jaccard's phenetic distance matrix based on a multi locus enzyme electrophoresis on 12 polymorphic loci. Reference stocks SO34 c14 (clonet 20) and MN (clonet 39) are zymodemes 1 and 11 respectively. 
TABLE II

Multilocus genotypes of the 20 zymodemes of Trypanosoma cruzi isolated from Bolivian vectors and identified with 13 polymorphic loci

\begin{tabular}{|c|c|c|c|c|c|c|c|c|c|c|c|c|c|c|c|}
\hline Zymodemes & $\begin{array}{c}\text { Number of } \\
\text { stocks }\end{array}$ & Host & G6pd & $G p i$ & Gd Nad & $G d N a d p$ & Got & $I d h$ & $M d h$ & Me 1 & Me 2 & Pep 1 & Pep 2 & Pgm & $6 P g d h$ \\
\hline 1 & 14 & T. infestans & 2 & 3 & 4 & 2 & 2 & 1 & 1 & 2 & 1 & 2 & 2 & 1,4 & 2,3 \\
\hline 2 & 3 & T. infestans & 2 & 3 & 4 & 2 & 2 & 1 & 1 & 1 & 1 & 2 & 2 & 1,4 & 2,3 \\
\hline 3 & 1 & T. infestans & 2 & 2 & 4 & 2 & 2 & 1 & 1 & 1 & 1 & 2 & 2 & 1,4 & 2,3 \\
\hline 4 & 6 & T. infestans & 2 & 3 & 4 & 2 & 2 & 1 & 1 & 2 & 1 & 2 & 2 & 1,4 & 3 \\
\hline 5 & 1 & T. infestans & 2 & 3 & 4 & 2 & 2 & 1 & 1 & 1 & 1 & 2 & 1 & 1,4 & 2,3 \\
\hline 6 & 1 & T. infestans & 2 & 3 & 4 & 2 & 2 & 1 & 1 & 1 & 1 & 2 & 1 & 1,4 & 3 \\
\hline 7 & 1 & T. infestans & 2 & 3 & 4 & 2 & 2 & 1 & 1 & 2 & 1 & 3 & 3 & 1,4 & 3 \\
\hline 8 & 1 & T. infestans & 2 & 3 & 3 & 2 & 2 & 1 & 1 & 1 & 1 & 2 & 2 & 1,4 & 3 \\
\hline 9 & 3 & R. pictipes & 2 & 3 & 4 & 2 & 2 & 1 & 1 & 2 & 1 & 1 & 4 & 1,4 & 3 \\
\hline 10 & 1 & T. infestans & 2 & 3 & 4 & 2 & 2 & 1 & 1 & 2 & 1 & 3 & 3 & 1,4 & 3 \\
\hline 11 & 1 & T. infestans & 1 & 1,2 & 2 & 2 & 1 & 2 & 1 & 1 & 2 & 4 & 3 & 2,5 & 1,3 \\
\hline 12 & 9 & T. infestans & 1 & 1,2 & 2 & 2 & 1 & 2,3 & 1 & 1 & 2 & 4 & 3 & 2,5 & 1,3 \\
\hline 13 & 1 & T. infestans & 1 & 1,2 & 2 & 2 & 1 & 2,3 & 1 & 1 & 2 & 4,5 & 3 & 2,5 & 1,3 \\
\hline 14 & 1 & T. infestans & 1 & 1,2 & 2 & 2 & 1 & 2,3 & 1 & 1 & 2 & 3 & 3 & 2,5 & 1,3 \\
\hline 15 & 1 & T. infestans & 1 & 1,2 & 2 & 1 & 1 & 2,3 & 1 & 1 & 2 & 4 & 3 & 2,5 & 1,3 \\
\hline 16 & 1 & T. infestans & 1 & 1,2 & 2 & 2 & 1 & 2,3 & 1 & 1 & 2 & 4 & 2 & 2,5 & 1,3 \\
\hline 17 & 1 & T. infestans & 1 & 1,2 & 2 & 2 & 1 & 2 & 1 & 2 & 2 & 4 & 3 & 2,5 & 1,3 \\
\hline 18 & 1 & T. infestans & 1 & 1,2 & 2 & 1 & 1 & 2 & 1 & 2 & 2 & 4 & 3 & 2,5 & 1,3 \\
\hline 19 & 1 & T. infestans & 2 & 2 & 1 & 2 & 1 & 2 & 1 & 1 & 2 & 4 & 3 & 1,4 & 3 \\
\hline 20 & 1 & T. infestans & 2 & 2 & 2 & 2 & 1 & 2 & 1 & 1 & 2 & 5 & 3 & $2,3,6$ & 3 \\
\hline
\end{tabular}


reference stock. All the stocks in which DNA was only recognized by probe 20 , belonged to $\mathrm{Z} 1$ through Z6, which together integrate the same sub-division of the first group (upper part of the dendrogram, Figure). Similarly, the stocks in which DNA was only recognized by probe 39 belonged to Z11 through Z18 and appeared clustered together in another subdivision belonging to the second group (lower part of the dendrogram). The other stocks unrecognized by the two probes were zymodemes Z7 through Z10, Z19 and Z20. The hybridization patterns of the isolated stocks correlated with the general topology of the dendrogram (Figure).

Three stocks presented a positive hybridization with the two probes (Table I), but in these cases the hybridization was intense with only one of the probes, and the zymodeme identified corresponded to the major clonet.

\section{DISCUSSION}

Natural mixtures of T. cruzi clones were previously evidenced by observation of mixed isoenzyme profiles and by isolation of different zymodemes from a single host (Tibayrenc et al. 1986, Brenière et al. 1989). More recently, the direct identification of $T$. cruzi in fecal samples by clonet specific DNA probes, showed that population mixtures in Bolivian T. infestans vectors are more frequent (Bosseno et al. 1996) than those detected by isoenzyme typing (10\%). The current work extends this result and demonstrates that clonet 20 is preferentially selected over clonet 39 from an heterogeneous population during culture. Previous works clearly established that clones have different growth rates in culture and according to computer models, rapid clonal selection should occur from mixed populations (Engel et al. 1982, Dvorak 1984). Laurent et al. (1997) compared groups of clones belonging to clonet 20 and 39 and showed that those of clonet 20 have a faster growth rate in monophasic LIT medium. This is in full agreement with our results and explains the selection of clonet 20 when the two clonets are present in the vector. Pinto Da Silva et al. (1998) studied experimental infections of T. infestans with mixtures of different genotypes; after vector infection, each stock was isolated and cultured for further genetic characterization by isoenzymes typing and RAPD. The authors observed the maintenance of the mixtures in the majority of cases. This result is in apparent contradiction with ours, however the authors reported that the clonal genotype 39 (corresponding to clonet 39 ), mainly appeared in trace quantities when it was initially mixed with clonal genotype 20 . Indeed this discrepancy could depend on culture time and it is worth noting that in the present work, all stocks were characterized after at least one month of sub- culture. This long lasting culture step should explain the drastic selection observed.

T. cruzi consists of a pool of clonal populations circulating among humans, domestic and sylvatic vectors and mammalian reservoirs, which have evolved in a great diversity with distinct biological, immunological and biochemical characteristics (Dvorak 1984, Sanchez et al. 1990, Andrade 1990). Identification of strains or clones is indispensable in order to study the variable characteristics of the populations. Genetic intrinsic characteristics at the molecular level which are not influenced by handing of strains in the laboratory, are the most suitable for identification. Nowadays the most usual methods are isoenzyme electrophoresis and genomic DNA analysis (Miles \& Cibulskis 1986, Morel et al. 1980, 1986, Tibayrenc \& Ayala 1988, Steindel et al. 1993, Brisse et al. 1998). Genotypedependent differences have been identified in relation to biological characters, and these results are a clear demonstration of the practical importance of genetic characterization of populations (Gonzalez et al. 1995, Laurent et al. 1997, Revollo et al. 1998). The current results show that during the classical culture procedure, one of the major clonets circulating in Bolivia is preferentially selected, demonstrating that direct identification of populations is necessary in order to avoid culture selection biases. In contrast, the direct identification failed to identify the clonal heterogeneity of the samples in $16 \%$ of the cases: e.g. clonet 20 alone was detected in seven feces samples before culturing, but in two stocks isolated from these feces after culture, clonet 39 alone or associated with clonet 20 were detected. This confirms that the natural populations of $T$. cruzi are frequently composed of a pool of clonal populations. In both cases, before and after culture, the clones were detected by PCR, a very sensitive technique; this implies that the absence of detection of a particular clone corresponds to the absence of this clone in the sample, or either its presence is in a very low quantity to be detected by PCR. In several cases the direct identification of clones in the feces before culture, failed to detect clones which were in a very low quantity, therefore, we cannot exclude the intervention of a natural selection that might take place in the digestive tract of the insect. Possibly, each life medium of the parasite interacts differently over the pool of the natural clonal population, and this helps to maintain the large clonal diversity observed in the $T$. cruzi taxon.

Several problems must be solved to develop the direct genetic characterization of $T$. cruzi in biological samples: (1) a high sensitive detection is needed because parasites are scarce in mammalian blood; (2) appropriate genetic markers presenting 
a variability linked with genetic clustering must be used; (3) the development of the new tools must be adapted to the geographical diversity of the $T$. cruzi genotypes and (4) the techniques must be easily applied to large sampling for further statistical interpretation. The correlation obtained between the clustering topology of the isolated stocks and the hybridization patterns of HVRm kDNA-PCR products (Figure) confirms and extends the interest of the HVRm regions for direct genetic identification of stocks. Nevertheless, the domestic Bolivian cycle may present a particular profile of the genetic diversity of $T$. cruzi clones. A similar approach in distinct endemic areas using kDNA probes, must be based on previous genetic diversity studies of local $T$. cruzi populations in order to evaluate genetic relationships between stocks and to be able to provide a genetic base to develop DNA probes.

\section{REFERENCES}

Andrade SG 1990. Influence of Trypanosoma cruzi strain on the pathogenesis of chronic myocardiopathy in mice. Mem Inst Oswaldo Cruz 85: 17-27.

Avila HA, Sigman DS, Cohen LM, Millikan RC, Simpson L 1991. Polymerase chain reaction amplification of Trypanosoma cruzi kinetoplast minicircle DNA isolated from whole blood lysates: diagnosis of chronic Chagas' disease. Mol Biochem Parasitol 48: 211-222.

Bastrenta B, Bosseno MF, Barnabé C, Tibayrenc M, Brenière SF 1999. Restriction fragment length polymorphism of $195 \mathrm{bp}$ repeated satellite DNA of Trypanosoma cruzi supports the existence of two phylogenetic groups. Mem Inst Oswaldo Cruz 94: 323328.

Ben Abderrazak S, Guerrini F, Mathieu-Daudé F, Truc P, Neubauer K, Lewicka K, Barnabé C, Tibayrenc M 1993. Methods in Molecular Biology, Human Press, Totowa, NJ, p. 361-382.

Bosseno MF, Telleria J, Vargas F, Yaksic N, Noireau F, Morin A, Brenière SF 1996. Trypanosoma cruzi: study of the distribution of two widespread clonal genotypes in Bolivian Triatoma infestans vectors shows a high frequency of mixed infections. Exp Parasitol 83: 275-282.

Brenière SF, Bosseno MF, Revollo S, Rivera MT, Carlier Y, Tibayrenc M 1992. Direct identification of Typanosoma cruzi natural clones in vectors and mammalian hosts by polymerase chain reaction amplification. Am J Trop Med Hyg 46: 335-341.

Brenière SF, Bosseno MF, Telleria J, Bastrenta B, Yaksic N, Noireau F, Alcazar JL, Barnabé C, Wincker P, Tibayrenc M 1998. Different behavior of two Trypanosoma cruzi major clones: transmission and circulation in Bolivian young patients. Exp Parasitol 89: 285-295.

Brenière SF, Bosseno MF, Telleria J, Carrasco R, Vargas F, Yaksic N, Noireau F 1995. Field application of PCR diagnosis and strain typing of Trypanosoma cruzi in Bolivian triatomines. Am J Trop Med Hyg 53: 179-184.
Brenière SF, Carrasco R, Revollo S, Aparicio G, Desjeux P, Tibayrenc M 1989. Chagas disease in Bolivia: clinical and epidemiological features and zymodeme variability of Trypanosoma cruzi strains isolated from patients. Am J Trop Med Hyg 41: 521-529.

Brisse S, Barnabé C, Tibayrenc M 1998. Trypanosoma cruzi: how many relevant phylogenetic subdivisions are there? Parasitol Today 14: 178-179.

Dvorak JA 1984. The natural heterogeneity of Trypanosoma cruzi: biological and medical implications. $J$ Cell Biochem 24: 357-371.

Engel JC, Dvorak JA, Segura E, Crane MS 1982. Trypanosoma cruzi: biological characterization of 19 clones derived from two chronic chagasic patients. I. Growth kinetics in liquid medium. J Protozool 29: 555-560.

Gonzalez J, Muñoz S, Ortiz S, Anacona D, Salgado S, Galleguillos M, Neira I, Sagua H, Solari A 1995. Biochemical, immunological, and biological characterization of Trypanosoma cruzi populations of the Andean North of Chile. Exp Parasitol 81: 125-135.

Jaccard P 1908. Nouvelles recherches sur la distribution florale. Bul Soc Vaudoise Sci Nat 44: 223-270.

Laurent JP, Barnabé C, Quesney V, Noel S, Tibayrenc M 1997. Impact of clonal evolution on the biological diversity of Trypanosoma cruzi. Parasitology 114: 213-218.

Miles MA, Cibulskis RE 1986. Zymodeme characterization of Trypanosoma cruzi. Parasitol Today 2: 94-98.

Morel C, Chiari E, Camargo E, Mattei D, Romanha A, Simpson L 1980. Strains and clones of Trypanosoma cruzi $i$ an be characterized by restriction endonuclease fingerprinting of kinetoplast DNA minicircles. Proc Natl Acad Sci USA 77: 6810-6814.

Morel CM, Deane MP, Goncalves AM 1986. The complexity of Trypanosoma cruzi populations revealed by schizodeme analysis. Parasitol Today 2: 4-8.

Nunes LR, de Carvalho MR, Buck GA 1997. Trypanosoma cruzi strain partition into two groups based on the structure and function of the spliced leader RNA and rRNA gene promoters. Mol Biochem Parasitol 86: 211-224.

Pinto Da Silva A, de Lana M, Bastrenta B, Barnabé C, Quesney V, Noel S, Tibayrenc M 1998. Compared vectorial transmissibility of pure and mixed clonal genotypes of Trypanosoma cruzi in Triatoma infestans. Parasitol Research 84: 348-353.

Revollo S, Oury B, Laurent JP, Barnabé C, Quesney V, Carrière V, Noel S, Tibayrenc M 1998. Trypanosoma cruzi: impact of clonal evolution of the parasite on its biological and medical properties. Exp Parasitol 89: 30-39.

Sanchez G, Wallace A, Olivares N, Diaz N, Aguilera X, Apt W, Solari A 1990. Biological characterization of Trypanosoma cruzi zymodemes: in vitro differentiation of epimastigotes and infectivity of culture metacyclic trypomastigotes to mice. Exp Parasitol 71: 125-133.

Sneath PHA, Sokal RR 1973. The Principle and Practice of Numerical Classification, Freeman, San Francisco, $537 \mathrm{pp}$.

Souto RP, Fernandes O, Macedo C, Campbell DA, 
Zingales B 1996. DNA markers define two major phylogenetic lineages of Trypanosoma cruzi. Mol Biochem Parasitol 83: 141-152.

Steindel M, Diaz Neto E, Menezes C, Romanha A, Simpson AJG 1993. Random amplified polymorfic DNA analysis of Trypanosoma cruzi strains. Mol Biochem Parasitol 60: 71-80.

Tibayrenc M 1995. Population genetics of parasitic protozoa and other microorganisms. Adv Parasitol 36: 47-115.

Tibayrenc M 1998. Genetic epidemiology of parasitic protozoa and other infectious agents: the need for and integrated approach. Int J Parasitol 28: 85-104.

Tibayrenc M, Ayala FJ 1988. Isoenzyme variability of Trypanosoma cruzi, the agent of Chagas' disease: genetical, taxonomical and epidemiological significance. Evolution 42: 277-292.

Tibayrenc M, Hoffmann A, Poch O, Echalar L, Le Pont F, Lemesre JL, Desjeux P, Ayala FJ 1986. Additional data on Trypanosoma cruzi isoenzymic strains encountered in Bolivia domestic transmission cycles. Trans $R$ Soc Trop Med Hyg 80: 442-447.

Thioulouse J 1989. Statistical analysis and geographical display of multivariate data on Machintoch. Computer Apply Biosciences 5: 287-292.

Veas F, Brenière SF, Cuny G, Brengues C, Solari A, Tibayrenc M 1991. General procedure to construct highly specific kDNA probes for clones of Trypanosoma cruzi for sensitive detection by Polymerase Chain Reaction. Cell Mol Biol 37: 73-84. 
608 Culture Selection of T. cruzi Clones - Marie-France Bosseno et al. 